\title{
A Model Maintenance Tool - Moving Forward with an Investment in a System-Wide Model
}

\author{
Derek Wride, Ralph Johnstone, Rodney Moeller, Carl Chan \\ and Joe Koran
}

In 2000, the Metropolitan Sewer District of Greater Cincinnati (MSDGC) initiated the development of a system-wide computer model (SWM) of their wastewater collection system to assist the agency in the assessment of the hydraulic performance of its system and in the prioritization of short- and long-term system improvements. The 42,000-node SWM was developed and calibrated, using EPA SWMM 4 (Huber, 1988), over a three-year period and represents over 1,500 miles of pipe (CDM, 2003).

In response to Consent Decree requirements (United States of America, $2002 \& 2003$ ), the SWM was used to perform a comprehensive hydraulic capacity assessment of the wastewater collection system under both dry- and wet-weather flow conditions and is currently being applied to find solutions to assure system capacity. The SWM has been integrated into the agency operations and is being applied to meet a variety of objectives, some of which will extend well into the future.

The SWM was built primarily with data from the Cincinnati Area Geographic Information System (CAGIS), a consortium of public and private entities with the goal of developing and maintaining infrastructure inventories in a common framework, in which MSDGC has participated since 1989. The inventory of sewer data in CAGIS has been diligently

Wride, D., R. Johnstone, R. Moeller, C.C. Chan and J. Koran. 2006. "A Model Maintenance Tool Moving Forward with an Investment in a System-Wide Model." Journal of Water Management Modeling R225-01. doi: 10.14796/JWMM.R225-01.

(C) CHI 2006 www.chijournal.org ISSN: 2292-6062 (Formerly in Intelligent Modeling of Urban Water Systems. ISBN: 0-9736716-2-9) 
maintained by MSDGC over the years. Weekly updates, including additions of new information and modifications to existing data, have ensured accurate, up-to-date representation of the existing sewer network. It is important that the SWM reflect the updated sewer data in order to assure that the proposed rehabilitative solutions identified by using the model properly reflect field conditions. This will help reduce the risk of over- or underdesigning system improvements and lead to more cost-effective planning.

A comparison between recent CAGIS sewer data and SWM data showed that approximately six thousand sewers have at least one attribute that is different. The large number of differences to review led to the development of a tool that will assist with the maintenance of the SWM. The tool compares the most up-to-date CAGIS data with current SWM data, documents the differences, allows for visualization of where the differences are located, provides a mechanism to methodically evaluate the differences and decide whether to incorporate the changes in the updated model, documents the decision making process so that it is available for subsequent maintenance iterations, and creates updated model data formatted for the model input files. This chapter presents the Cincinnati SWM Maintenance Tool in its first development phase, including the results from the comparison task highlighting the need for a tool, the logic behind the tool, and its functionality.

\subsection{The Need}

\subsubsection{An Investment}

Any wastewater collection system inevitably changes, to some degree, over time. Pipes, manholes, and system appurtenances are replaced, upsized, realigned, re-lined, abandoned, or deteriorated due to aging of the infrastructure. Modifications to the collection system occur primarily as part of a regular MSD Operations and Maintenance (O\&M) program and Capital Improvement Program (CIP). The collection system changes are regularly updated in CAGIS. MSD has dedicated staff to track the changes to the system and coordinate with CAGIS to update the data on a weekly basis.

The SWM was created and calibrated to a certain point in time. When the SWM is used for MSD applications, changes to the collection system need to be assessed to determine the potential impact on the model application. When a model update occurs, an update time is established 
which indicates that the modeled collection system will include changes to the system up to that time based on the best available data. It is important to note that the SWM is built primarily on CAGIS data and may not exactly represent the collection system as it exists in the field according to the update time, but represents the collection system based on CAGIS's representation. Simply put, the model representation of the collection system is only as accurate as CAGIS is up-to-date.

With the collection system changing constantly, the CAGIS data changing on a weekly basis, and the model representing a specific time period, the challenge that has been set in motion is how and when to update the model to prevent it from becoming too outdated. The risk in allowing the model to become too outdated is that it becomes less reliable as a tool to help study system improvements and changes. The results from an outdated model may be inaccurate because the model does not accurately represent field conditions. This can lead to the potential under- or over-design of system improvements. The under- or over-design of system facilities could amount to millions of dollars when viewed in light of the US\$1.5 billion consent decree mandate to fix wet-weather problems over the next seventeen years (through 2022). Maintaining the SWM up-to-date will lead to greater reliability and accuracy in the use of the modeling tool and yield financial and regulatory benefits for which the investment in a SWM was intended.

\subsubsection{Differences between SWM and CAGIS}

An initial comparison of the SWM data to recent CAGIS data was performed to help assess the level of model updating needed. The effort only compared pipe data between the model input files and the most recent CAGIS data. The SWM input files were created in essentially two phases; the first phase was the development of the model corresponding to the Mill Creek Basin, which is the largest of the three major drainage basins. Phase two developed the portions of the SWM corresponding to the Little Miami and Great Miami drainage basins. The Mill Creek model data was based on 2001 CAGIS data while the Little and Great Miami Basins were created based on 2002 CAGIS data. The CAGIS data used for the SWM Maintenance Tool project and for the comparison to the SWM data was from late 2004.

Table 1.1 shows the results of the initial comparison of the pipe attribute data between the SWM and the recent CAGIS data. The approximately 
6,000 differences resulting from the initial comparison clearly point to the need for a tool to help in the model update task.

Table 1.1 Number of differences between SWM and recent CAGIS.

\begin{tabular}{ccccccc}
\hline $\begin{array}{c}\text { Year } \\
\text { Changed }\end{array}$ & $\begin{array}{c}\text { Upstream } \\
\text { Invert }\end{array}$ & $\begin{array}{c}\text { Downstream } \\
\text { Invert }\end{array}$ & $\begin{array}{c}\text { Pipe } \\
\text { Size }\end{array}$ & $\begin{array}{c}\text { Pipe } \\
\text { Shape }\end{array}$ & $\begin{array}{c}\text { Pipe } \\
\text { Length }\end{array}$ & $\begin{array}{c}\text { TOTAL } \\
\text { Differences }\end{array}$ \\
\hline 2001 & 150 & 83 & 74 & 0 & 58 & 365 \\
2002 & 533 & 630 & 268 & 0 & 203 & 1,634 \\
2003 & 458 & 488 & 105 & 4 & 116 & 1,171 \\
2004 & 602 & 636 & 90 & 3 & 154 & 1,485 \\
no date & 492 & 574 & 258 & 0 & 285 & 1,609 \\
\hline TOTAL & 2,235 & 2,411 & 795 & 7 & 816 & $\mathbf{6 , 2 6 4}$ \\
\hline
\end{tabular}

Of the 6,264 differences, the final number that will be included in the model update will be much smaller for a number of reasons, but they still represent the number of items that need to be reviewed. The actual number of items to be updated has not yet been determined as the update decision for each difference has not been completed at this point. However, a review of the initial comparison results points to the following four primary reasons for having to update a much smaller number than the approximate 6,000 items listed.

1. This initial comparison represents the results from the first system-wide comparison between the SWM and CAGIS since the creation of the SWM. This covers approximately three years, while future updates are anticipated to occur more frequently,

2. There has been increased activity to update MSD data in CAGIS over the last few years so it will be more up-to-date to begin with for subsequent model updates.

3. Differences in the attribute characteristics may be insignificant and would not merit the effort to include in a model update.

4. Some differences are acceptable because a relatively small percentage of SWM data is based on sources 
other than CAGIS, such as the field investigation program specific to the SWM development.

The initial comparison includes two other categories not listed in Table 1.1:

- pipes that are in CAGIS that meet the SWM size criteria, but are not in the SWM, and

- $\quad$ pipes that are in the SWM, but not in CAGIS.

For the former category, most of these have already been reviewed as part of the development of the SWM and have been excluded for reason. These pipes will be flagged appropriately and will not show up on the list of differences for future model updates (unless the date in the "Date Modified" field is more recent than the last update). For the latter, some pipes were merged in the SWM to improve the model stability. These pipes will also be flagged and excluded from the differences list for subsequent model updates.

Figure 1.1 illustrates the location of the differences across the MSD service area as represented by the SWM. The magnitude and widespread distribution of differences (dark marks across all three basins) support the need for a model update tool.

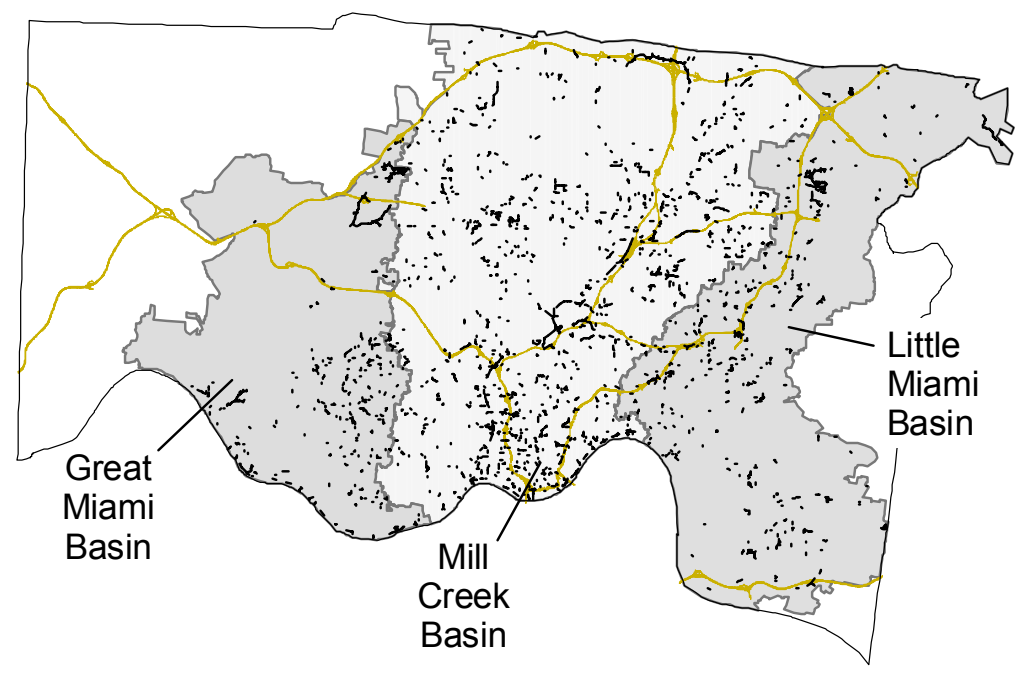

Figure 1.1 Pipes with differences in attributes (dark marks across all 3 basins) between SWM (2001) and CAGIS (2004). 


\subsection{The Tool}

The SWM Maintenance Tool is anticipated to be developed in a series of phases to provide a tool sufficient to meet immediate needs and to allow for flexibility to adapt the tool to meet future needs, such as major changes in both the agency modeling (U.S.EPA, 2002 and Rossman, 2003) and GIS software, which are anticipated, as well as other potential changes. This chapter discusses the initial phase of the tool development.

\subsubsection{Logic}

The SWM Maintenance Tool comprises two components: an Access database and an Arcview ${ }^{\mathrm{TM}}$ extension. The database format uses existing software familiar to the agency, and provides relatively easy customization and compatibility with other database tools developed for the SWM.

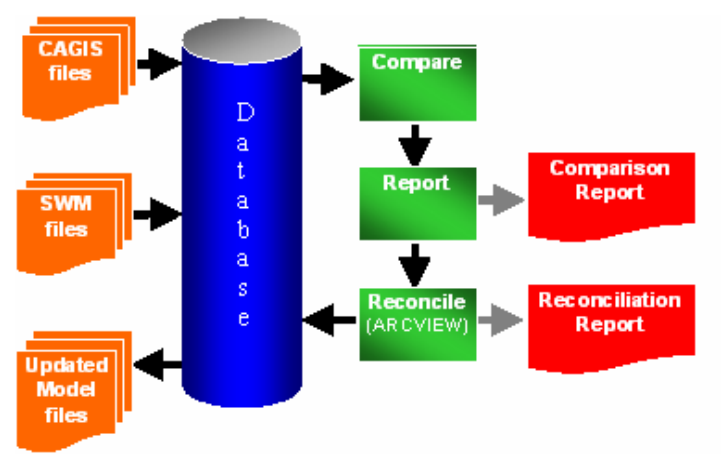

Figure 1.2 The SWM Maintenance Tool logic diagram.

The Arcview extension takes advantage of in-house GIS capabilities, enabling quick and efficient implementation of the tool within the agency. Between the two components, the SWM Maintenance Tool:

1. provides a central location for managing the model data,

2. compares the SWM data to CAGIS data,

3. identifies and documents the differences between the SWM and CAGIS,

4. uses GIS to visualize the location of the differences, 
5. provides a mechanism to methodically review differences and decide whether to include them in the update,

6. documents the decision making process so it is available for subsequent update iterations, and

7. creates updated, properly formatted model input data.

\subsubsection{Tool Component - Access Database}

The Access database component provides for items 1, 2, 3, 6, and 7 of the seven tool capabilities outlined above in section 1.2.1. The database component serves primarily as a repository of the SWM data and provides a convenient means to perform various functions on the data. Figure 1.3 illustrates the interface to the database and the five primary buttons (labeled A through E) used to perform a unique step in the process. The purpose of each button will be briefly described.

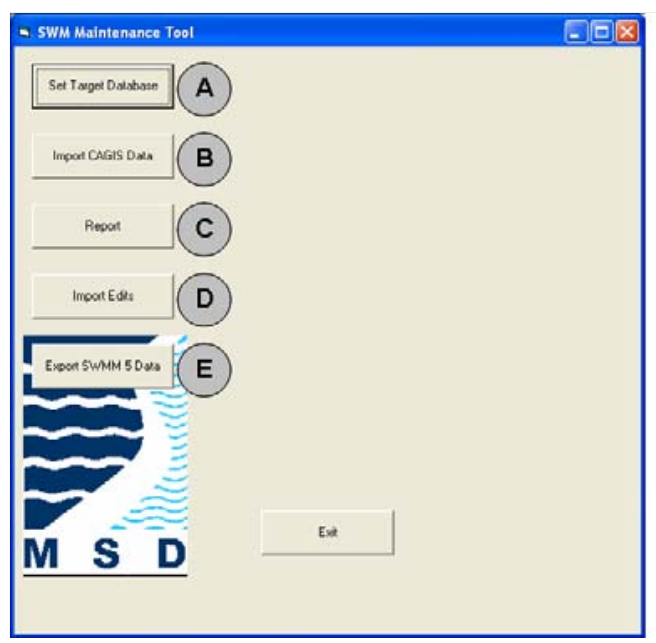

Figure 1.3 The SWM Maintenance Tool database interface.

Set Target Database (A)

The button labeled "Set Target Database" enables the user to set the location to tell the system where the master database is located. This database is a Microsoft Access file which contains the model data tables and a few 
support tables. Once the target database has been set, the SWM

Maintenance Tool form will display the name of the target database next to the "Set Target Database" button.

Import CAGIS Data (B)

The button labeled "Import CAGIS Data" enables the user to import the latest CAGIS dBase files for the sewer manholes and pipes into the target database. The user will be prompted for a "date stamp" for the CAGIS data being imported. This date stamp will become part of the table names in the reporting process. Upon CAGIS import, the system creates two new tables based on the selection criteria for modeling. First, it selects all sanitary and separate pipes that are 12 inches $(305 \mathrm{~mm})$ and greater from the CAGIS pipes table. Then it adds all combined pipes 18 inches $(460 \mathrm{~mm})$ and greater to the selection. These represent the SWM pipe size criteria. Then it adds all pipes that do not meet the selection criteria, but are in the modeled pipes table anyway, to the selection. It then creates a new CAGIS pipes table from this selection. Finally, it creates a new CAGIS manholes table consisting of all of the manholes connected to the selected pipes. After importing CAGIS data, the system logs the time and table names and other information.

Report (C)

The button labeled "Report" enables the user to create and export the tables of differences between the CAGIS data and the model data. The tables created are listed below with a brief description of its contents:

1. v_yyyy-mm-dd_DSInvDiffs.dbf - pipes with differences in downstream invert elevations

2. v_yyyy-mm-dd_LengthDiffs.dbf - pipes with differences in pipe lengths

3. v_yyyy-mm-dd_PipesInCagisNotInModel.dbf - pipes that are in CAGIS that meet the SWM pipe size criteria, but are not in the SWM

4. v_yyyy-mm-dd_PipesInModelNotInCagis.dbf - pipes that are in the SWM, but are not in CAGIS

5. v_yyyy-mm-dd_ShapeDiffs.dbf - pipes with differences in pipe shapes

6. v_yyyy-mm-dd_SizeDiffs.dbf - pipes with differences in pipe sizes

7. v_yyyy-mm-dd_SplitPipes.dbf - pipes in the SWM that are not in CAGIS, but where the upstream and downstream manholes match in CAGIS, but not on the same pipe 
8. v_yyyy-mm-dd_USInvDiffs.dbf - pipes with differences in upstream invert elevations

9. t_yyyy-mm-dd_COUNTS.dbf - provides a summary of the number of each of the records in each of the above tables

The "yyyy-mm-dd" designation is the CAGIS date-stamp supplied upon data import. Upon completion, the system logs the date of export and location of the exported tables.

Import Edits (D)

The "Import Edits" button is used after specifying your edit choices through the SWM Maintenance Arcview Extension (described in next section) and enables the user to import the edit choices made in the GIS environment. The choice of whether to include a difference between the SWM and CAGIS data as a change to the SWM is made in the GIS environment. The documentation of that decision is brought into the central database using this button where it will be available for subsequent update iterations. The system will read in the tables it exported in the previous step, and update the model data with the CAGIS data for each difference that was flagged with "Update". Upon completion of the Import Edits process, the date is logged.

Export SWMM 5 Data (E)

The "Import Edits" button enables the user to export SWMM 5 datasets to text files that can be incorporated into SWMM 5 input files. From a dropdown list, the user can select one of the seven sub-basins that correspond to the seven major MSD wastewater treatment plants. The location of the file can be selected by pressing the button next to the filename box and the output filename can be specified. The output text file includes full SWMM 5 input sections for the following data that are ready to be pasted into the appropriate sections of the SWMM 5 input files:

- conduits

- junctions

- storages

- outfalls

- $\mathrm{x}$-sections

- coordinates.

At each step of the model maintenance process using the SWM Maintenance Tool, the process history is documented with the use of a log file. The history of the steps taken are recorded and displayed in the SWM Maintenance Tool interface next to each button as illustrated in Figure 1-4. 


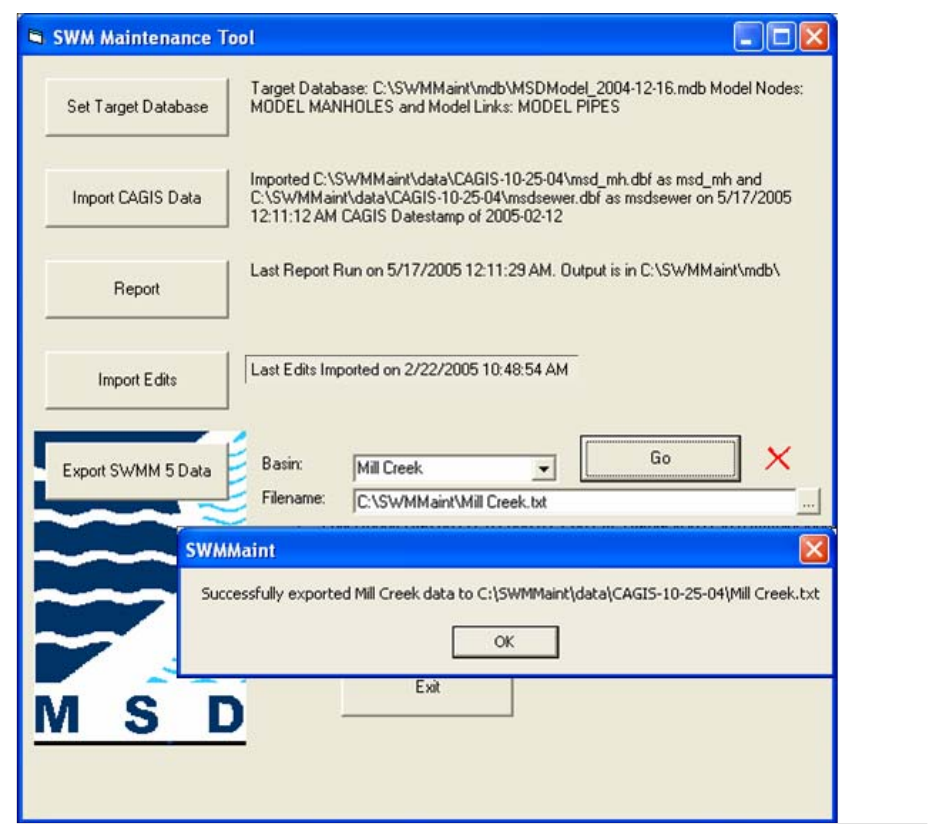

Figure 1.4 Documentation of the model update process using the database component of the SWM Maintenance Tool.

The documentation of the model update process is vital to subsequent model update iterations. The database component is central to the SWM Maintenance Tool and facilitates the task of updating the SWM. The GIS component offers complementary capability to further assist in the process.

\subsubsection{Tool Component - Arcview ${ }^{\mathrm{TM}}$ Extension}

The SWM Maintenance Extension is an Arcview 3.3 extension that is designed to be used in conjunction with the database component of the SWM Maintenance Tool. It provides visualization and documentation capabilities to the model update process. The Arcview environment is where the decisions are made as to whether to include a difference between the SWM and CAGIS into the model update. The use of the database component of the tool is a prerequisite to using the Arcview extension tool. 
The reporting process of the SWM Maintenance Tool outputs several tables in dBase format. Among these tables is what is referred to as "differences tables". These tables are the following:

1. v_yyyy-mm-dd_DSInvDiffs.dbf

2. v_yyyy-mm-dd_LengthDiffs.dbf

3. v yyyy-mm-dd PipesInCagisNotInModel.dbf

4. v_yyyy-mm-dd_ShapeDiffs.dbf

5. v_yyyy-mm-dd_SizeDiffs.dbf

6. v_yyyy-mm-dd_USInvDiffs.dbf

where "yyyy-mm-dd" is the CAGIS date-stamp provided by the user on table import.

The user must add these tables to an Arcview project and use the tools provided by this extension to flag each record with one of the following:

1. Update

2. Do Not Update

3. Do Not Update and Do Not Ask Again (until the CAGIS date-modified changes for this pipe)

The user may also input comments into a comments field for each record. After processing with this extension, the user returns to the database component of the SWM Maintenance Tool and uses the "Import Edits" button to bring the edit choices into the master database and update the model data with the CAGIS data where desired.

The capabilities of the customized Arcview extension are discussed below.

\section{Configure Tool}

Once the extension is activated in Arcview, the SWM Maintenance Extension adds one button to the Project button bar. This button is used to set the view, model pipe themes, model manholes theme, CAGIS pipes theme, CAGIS manholes theme, and current CAGIS date stamp of the data. This needs to be done once as the system will remember the choices made.

\section{Table Button Bar}

The SWM Maintenance Extension adds four buttons to the Table Button Bar in Arcview. Below is a description of the function of each.

1. Locates the selected pipe in the view.

2. Selects recent CAGIS modifications (CAGIS DATE MOD more recent than last maintenance).

3. Individual edit tool that iterates through selected records (or all if none selected) one at a time. 
4. Bulk edit tool that can set and edit choices to all selected (or all if none selected) records.

View Button Bar

The SWM Maintenance Extension adds one button to the View button bar. This button locates the selected pipe in one or more of the differences tables and zooms to that pipe.

\section{Configuration Button}

After pressing the Configuration button, the user will be prompted for several inputs the system needs to know in order to be able to link pipes in a table with the corresponding pipes in a view, and vice versa.

\section{CAGIS Date Stamp}

This button is used to enter the current CAGIS date stamp, which is used to find the current differences tables. The differences tables have the CAGIS date stamp in their names (e.g., v_2004-10-25_usinvdiffs.dbf.)

Locate Selected Pipe

This button enables the user to locate the selected pipe(s) in the view.

\section{Get Recent Modifications}

This button enables the user to automatically select all of the records where the CAGIS Date Modified is later than the last time the update process was run.

\section{SWM Maintenance Dialog}

This button enables the user to iterate through the selected records (all if none selected) and shows the SWM Maintenance Dialog for each record. Pressing the locate button zooms to the selected pipe. Pressing the "Next" button applies the selected option and automatically moves to the next record. The SWM Maintenance Dialog is illustrated in Figure 1.5.

\section{Bulk Processing}

This button enables the user to assign a single choice to all of the selected records (or all of the records if none is selected). Any comment entered, and the option selected by the user, will be applied to the entire set at once. This can speed up the model process where a group of pipes all require the same action. Figure 1.6 shows this capability in the SWM Maintenance Dialog. 


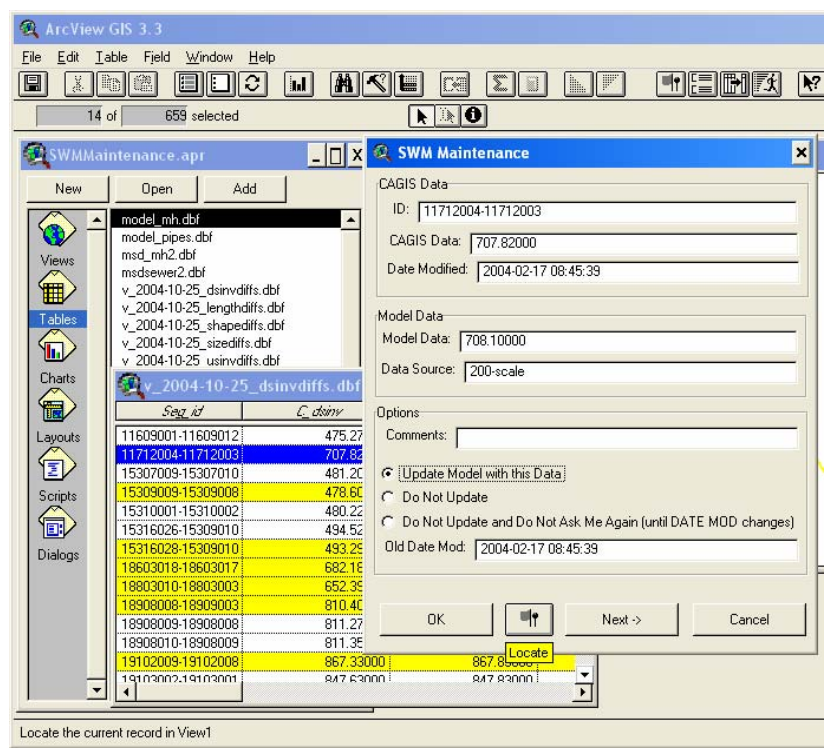

Figure 1.5 SWM maintenance dialog for making update decisions.

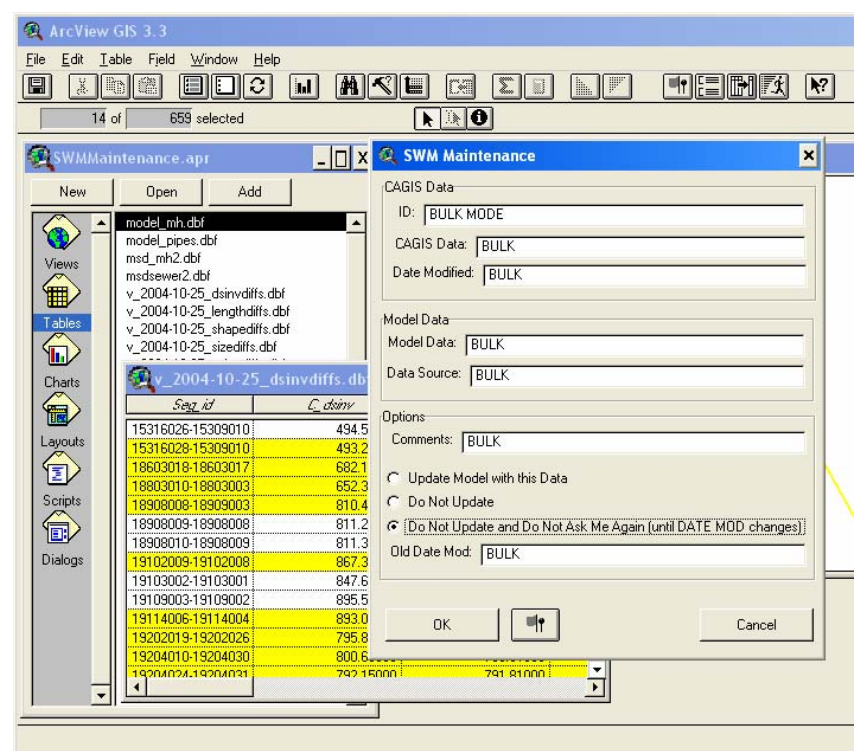

Figure 1.6 Bulk processing capability in the SWM maintenance dialog. 
Locate from View

The Locate from View button enables the user to locate the records in the differences tables that correspond with the pipe selected in the view. Figure 1.7 illustrates the structure of a "differences" table in Arcview.

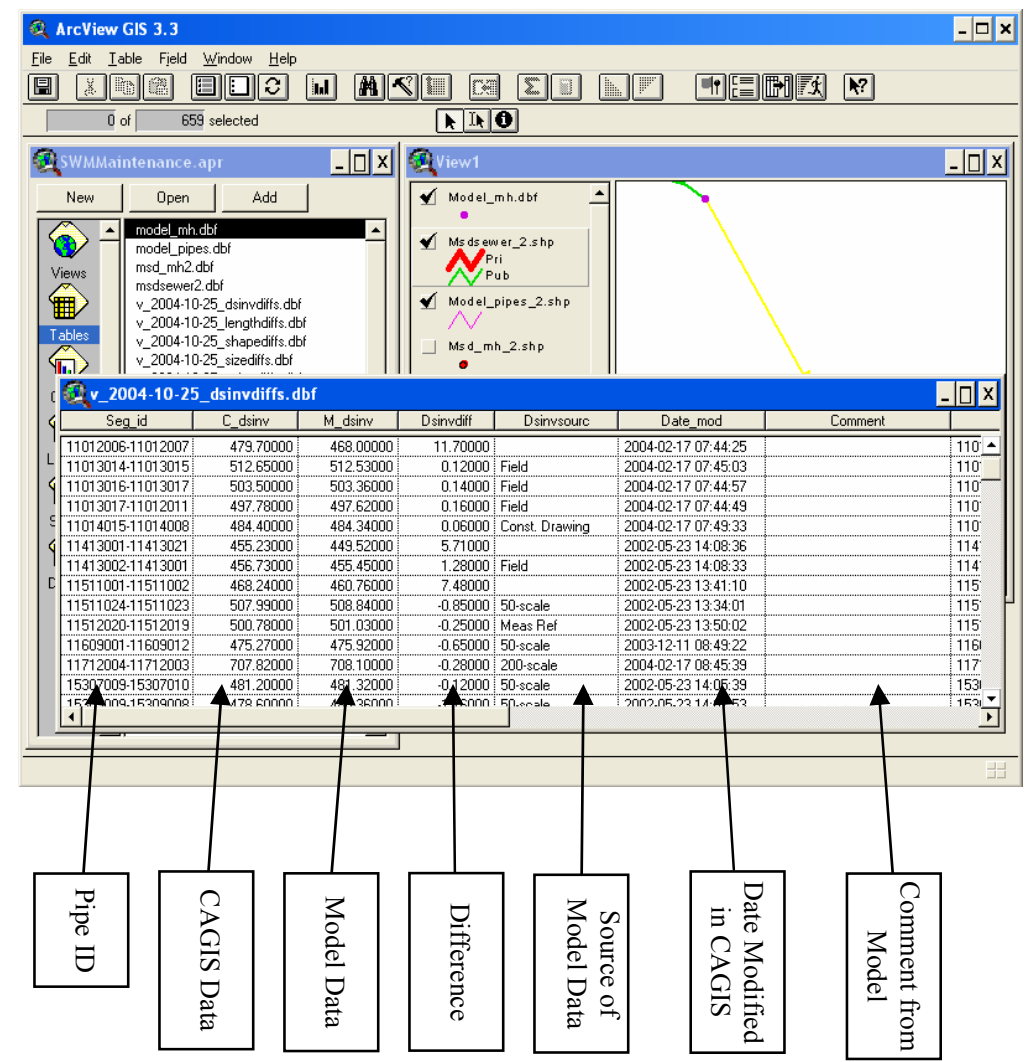

Figure 1.7 Structure of differences table in Arcview.

Figure 1-8 illustrates a continuation of the structure of a "differences" table in Arcview, where the decision making process is documented and imported back into the central Access database using the "Import Edits" button of the database tool. These decisions will be available for the subsequent model update. 


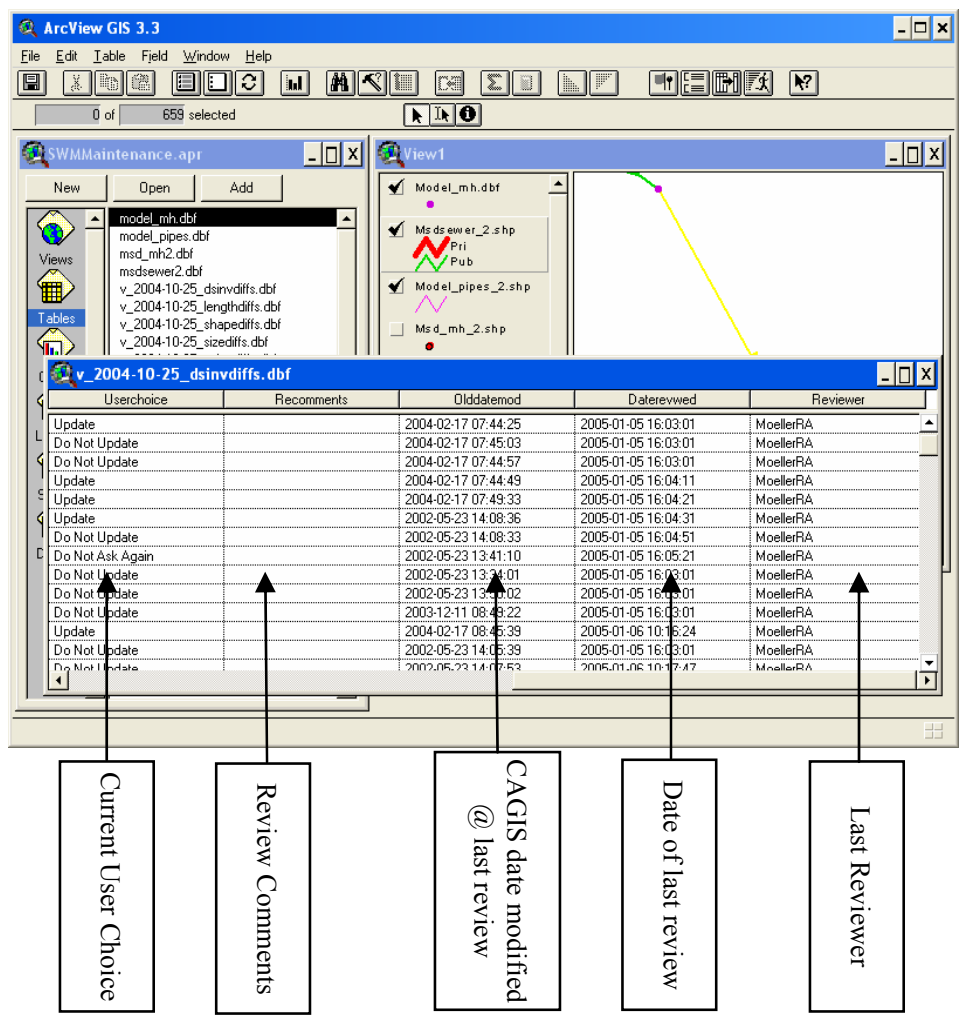

Figure 1.8 Documentation of decision making process in the differences table in Arcview.

\subsection{Summary}

The SWM Maintenance Tool was developed to assist with the potentially laborious task of updating the 42,000-node SWM. It will serve as an important tool to protect the investment MSD has made in a system-wide model. The choice to update the SWM over allowing it to continually outdate itself means it can be continually used to achieve the purpose for which it was created. The benefits of the SWM Maintenance Tool include:

- making the model maintenance task more efficient, which directly saves time and money, 
- providing the capability to perform model update iterations as often as desired,

- utilizing in-house GIS technology that enables the agency staff to quickly implement the tool with minimal training, and

- providing an updated SWM that can be used reliably and accurately, which will lead to greater costeffective planning.

As the SWM Maintenance Tool is implemented into the agency and model updates are performed to the SWM, refinements to the tool will occur and take advantage of its flexibility to adapt to the future needs of the agency in maintaining an up-to-date SWM. The SWM Maintenance Tool offers the capability to help protect their investment in a system-wide model well into the future.

\section{References}

CAGIS (Cincinnati Area Geographic Information System), http://cagis.hamiltonco.org/CAGIS/.

CDM, 2003. System Wide Model, Executive Summary. March 2003.

Huber, W. C. and R. E. Dickinson (1988). Storm Water Management Model - Version 4: User's Manual; Cooperative Agreement CR-811607; U.S. EPA; Athens, Georgia.

Rossman, L.A., R.E. Dickinson, T. Schade, C.C. Chan, E. Burgess, D. Sullivan and F. Lai. 2003. "SWMM 5 - the Next Generation of EPA's Storm Water Management Model." Journal of Water Management Modeling R220-16. doi: 10.14796/JWMM.R220-16.

U.S. EPA, 2002. SWMM Redevelopment Project Plan - Version 5; U.S.EPA Office of Research and Development - Water Supply and Water Resources Division; Cincinnati, Ohio; February 19, 2002.

United States of America and the State of Ohio, 2002. Interim Partial Decree on Sanitary Sewer Overflows. United States of America and The State of Ohio vs. the Board of County Commissioners of Hamilton County, Ohio and The City of Cincinnati. Civil Action No. 1:02cv00107.

United States of America, the State of Ohio, and the Ohio River Valley Water Sanitation Commission, 2003. Consent Decree on Combined Sewer Overflows, Wastewater Treatment Plants and Implementation of Capacity Assurance Program Plan For Sanitary Sewer Overflows. United States of America, The State of Ohio, and Ohio River Valley Water Sanitation Commission vs. The Board of County Commissioners of Hamilton County, Ohio and The City of Cincinnati. Civil Action No. C-1-02-107. 